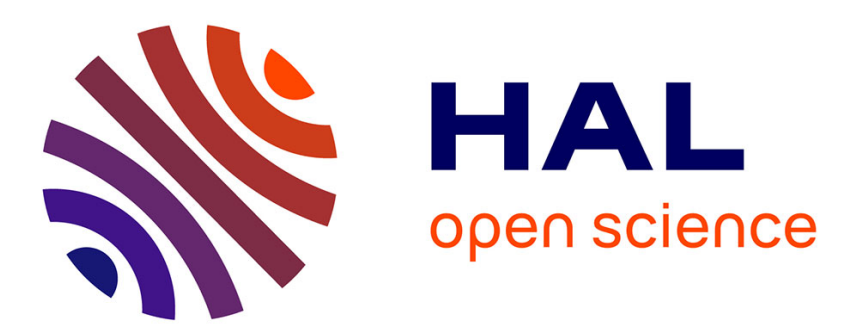

\title{
On the consideration of motion effects in the computation of impulse response for underwater acoustics inversion
}

Nicolas Josso, Cornel Ioana, Jerome I. Mars, Cedric Gervaise, Yann Stéphan

\section{- To cite this version:}

Nicolas Josso, Cornel Ioana, Jerome I. Mars, Cedric Gervaise, Yann Stéphan. On the consideration of motion effects in the computation of impulse response for underwater acoustics inversion. Journal of the Acoustical Society of America, 2009, 126 (4), pp.1739-1751. 10.1121/1.3203308 . hal-00422610

\author{
HAL Id: hal-00422610 \\ https://hal.science/hal-00422610
}

Submitted on 7 Oct 2009

HAL is a multi-disciplinary open access archive for the deposit and dissemination of scientific research documents, whether they are published or not. The documents may come from teaching and research institutions in France or abroad, or from public or private research centers.
L'archive ouverte pluridisciplinaire HAL, est destinée au dépôt et à la diffusion de documents scientifiques de niveau recherche, publiés ou non, émanant des établissements d'enseignement et de recherche français ou étrangers, des laboratoires publics ou privés. 


\title{
On the consideration of motion effects in the computation of impulse response for underwater acoustics inversion
}

\author{
Nicolas F. Josso, ${ }^{\text {a) }}$ Cornel loana, and Jérôme I. Mars \\ GIPSA-lab, Grenoble Institute of Technology (GIT), 961 rue de la Houille Blanche, 38402 St Martin \\ d'Hères, France \\ Cédric Gervaise \\ E3I2, EA3876, ENSIETA, Université Européenne de Bretagne, 2 rue Francois Verny, 29806 Brest Cedex, \\ France \\ Yann Stéphan \\ SHOM, CS52817, 13 rue du Chatellier 29228 Brest Cedex 2, France
}

(Received 14 January 2009; revised 7 July 2009; accepted 9 July 2009)

\begin{abstract}
The estimation of the impulse response (IR) of a propagation channel may be of great interest for a large number of underwater applications: underwater communications, sonar detection and localization, marine mammal monitoring, etc. It quantifies the distortions of the transmitted signal in the underwater channel and enables geoacoustic inversion. The propagating signal is usually subject to additional and undesirable distortions due to the motion of the transmitter-channel-receiver configuration. This paper shows the effects of the motion while estimating the IR by matched filtering between the transmitted and the received signals. A methodology to compare IR estimation with and without motion is presented. Based on this comparison, a method for motion effect compensation is proposed in order to reduce motion-induced distortions. The proposed methodology is applied to real data sets collected in 2007 by the Service Hydrographique et Océanographique de la Marine in a shallow water environment, proving its interest for motion effect analysis. Motion compensated estimation of IRs is computed from sources transmitting broadband linear frequency modulations moving at up to 12 knots in the shallow water environment of the Malta plateau, South of Sicilia.
\end{abstract}

(C) 2009 Acoustical Society of America. [DOI: 10.1121/1.3203308]

PACS number(s): 43.30.Pc, 43.60.Mn, 43.60.Pt, 43.30.Cq [AIT] Pages: 1739-1751

\section{INTRODUCTION}

The knowledge of the impulse response (IR) of propagation channels is potentially interesting for a large number of underwater acoustics applications such as underwater communication, sonar detection and localization, marine mammal monitoring, etc. The IR estimate is also central for geoacoustic inversion using matched impulse response (MIR) techniques. ${ }^{1}$ The most popular method to estimate IR is the so-called matched filtering, ${ }^{2}$ where the received signal is correlated with the transmitted one.

Ideally, with an additive white-noise background, the matched filtering operation processing correlates the received signal with time-delayed versions of the transmitted signal. When the motion of the transmitter and receiver is well monitored, as in the case of active ocean acoustic tomography, methods such as matched-field-processing. ${ }^{3}$ or MIR can take into account the motion effects, even if a highly computational cost may be required for broadband signals. When the motion of the transmitter and receiver is unknown (as it is the case for passive ocean acoustic tomography $\left.{ }^{4,5}\right)$, these methods cannot be applied any longer. In this paper, we propose a new method to estimate and

\footnotetext{
a) Author to whom correspondence should be addressed. Electronic mail: nicolas.josso@gipsa-lab.inpg.fr
}

compensate the motion effects. This work is a contribution to the development of a passive tomography system using transient signals.

When the motion of the transmitter-channel-receiver configuration is not known, the received signal could be correlated against a family of reference signals that represent as well all possible receptions. The set of reference signals would account for all the possible velocities of the configuration and the multipath propagation effects of the environment. For example, Qian and $\mathrm{Chen}^{6}$ and Mallat and Zhang ${ }^{7}$ proposed a matching pursuit algorithm on transitory signals, which adaptively decomposes any signal into a linear combination of best-matched basis functions that are selected from a dictionary of Gabor atoms. Zou et al. ${ }^{8}$ extended some earlier results on steady-motion based Dopplerlet transform and introduced the application of Dopplerlet transform to the estimation of range and speed of a moving source.

Not many works have been reported on the problem of solving the resulting wave equation for a moving source in an acoustic waveguide. Guthrie et al.,${ }^{9}$ Hawken, ${ }^{10}$ and more recently Lim and Ozard ${ }^{11}$ considered sources moving radially or horizontally and obtained expressions for the acoustics field using normal theory. Flanagan et al. ${ }^{12}$ and Clark et $a l .{ }^{13}$ formulated the moving source problem in terms of ray theory, where each raypath has different Doppler shift according to its angle of emission. Most solutions are given in 
terms of contemporary time, i.e., the time at which the sound reaches the receiver but Lim and Ozard expressed their solution in terms of retarded time, i.e., the time at which the sound was transmitted by the source.

This paper investigates the effects of the motion that often exists in an operating transmitter-channel-receiver configuration in geoacoustic inversion. Doppler effect consequences on the estimation of the IR are shown and explained for shallow water environments with matched filtering between the transmitted and received signals. The studied signals have very low central frequencies (around $1300 \mathrm{~Hz}$ ) and high bandwidth (around $2000 \mathrm{~Hz}$ ). For feasibility purposes, it is considered that the relative motion existing between the transmitter and the receiver is horizontal with a constant speed during transmissions.

The performance of the correlation receiver in delay and Doppler can be described with the ambiguity function. If the signal is narrowband, then the conventional formulation of the ambiguity function is appropriate. In this case, the effects of motion, which are a compression in time for approaching sources and an expansion for receding sources, are approximated as simple carrier-frequency shifts of the transmitted waveform. For the narrowband case, the correlation receiver has a reference set of signals composed by time-delayed and carrier-frequency-shifted versions of the transmitted signal. However, this is no longer valid when the ratio bandwidth under central frequency increases. In our study, a multipath wideband ambiguity function is introduced in order to account for a different broadband Doppler effect for each path. In the wideband case, the correlation receiver has reference signals that are time-delayed and time-scaled versions of the transmitted one. Hermand and Roderick ${ }^{14}$ fully described and formulated the narrowband and wideband ambiguity functions for active sonar systems. The interferences that can occur in the cases of reflection on multiple moving targets are also analyzed. The purpose of this paper is to analyze the broadband ambiguity function with the same approach as in Ref. 14 and apply it to the estimation of the IR of an underwater acoustic propagation channel when motion exists between the source and the receiver. We will show that when high bandwidth and very low central frequency signals are transmitted, the wideband ambiguity plane enables estimating and compensating the Doppler effects which modify the underwater acoustic propagation channel. Doppler effects will be shown to differ for each propagation path on simulated and real data.

This paper is organized as follows. Section II presents a transient signal modeling for a multipath environment with rectilinear, constant speed motion. Then Sec. III describes the motion effects both on the estimation of an IR computed with a correlation receiver process and on the narrowband and wideband ambiguity functions in a multipath environment. A Doppler effect estimation and removal technique on the IR and its applications on simulated data are presented in Sec. IV. The results on a real data set are presented in Sec. V. We close in Sec. VI with conclusions.

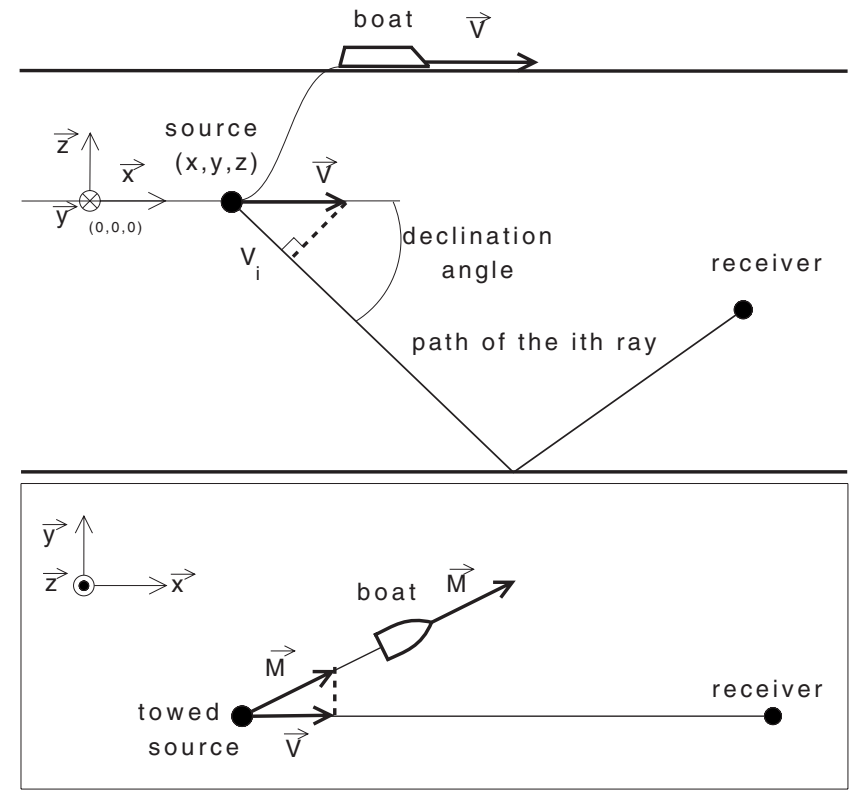

FIG. 1. Schematic illustration of how $v_{i}$ is computed from the motion vectors $\vec{M}$ and $\vec{v}$. The top panel represents a side view of the source-receiver configuration while the bottom panel is a top view. For simplicity, it is assumed here that the speed is constant.

\section{MODELING WAVE PROPAGATION AND MOVING TRANSIENT EMISSION}

The wideband Doppler effect in a multipath environment is presented in this section using contemporary time, i.e., the time at which the sound reaches the receiver, and retarded time, i.e., the time at which the sound was transmitted by the source considering a constant speed of propagation in the medium.

\section{A. Signal received for one ray}

In this section, we consider a fixed receiver and a source with constant speed motion $\vec{M}$. The source emits a signal for $T \mathrm{~s}$ while moving at a constant speed $v$ along the $x$ axis, as illustrated in Fig. 1. For one emission, the source position along the $x$ axis is

$$
x_{0}-\frac{v T}{2} \leq x \leq x_{0}+\frac{v T}{2},
$$

where $x_{0}$ is the position of the source after $T / 2 \mathrm{~s}$. We define a time axis $u$ for the emission, which is the retarded time, and $t$ for the reception, which is the contemporary time, following a relation of the form

$$
u+T_{i}(u)=t,
$$

where $T_{i}(u)$ refers to the time delay of the $i$ th ray and $u$ verifies

$$
-\frac{T}{2} \leq u \leq \frac{T}{2} .
$$

Relation (2) means that a signal transmitted at the delayed time $u$ is received on the $i$ th ray at the contemporary time $t$ equal to $u$ plus the propagation time along the $i$ th ray. If $L_{i}(u)$ 
is defined as the path length of the $i$ th ray in meters, formula (2) can be rewritten as

$$
u+\frac{L_{i}(u)}{c}=t,
$$

where $c$ is the speed of the sound in the medium. Assuming that the latter is constant, the path length of the $i$ th ray is defined as

$$
L_{i}(u)=\sqrt{x_{i}(u)^{2}+z_{i}^{2}},
$$

where $\left(x_{i}(u), z_{i}\right)$ represents the position of the virtual source from which the signal propagating along the $i$ th ray seems to have been radiated (the expressions of $z_{i}$ are given in Appendix A for all rays). It is worthy noting that each of the virtual sources seems to move at a different apparent speed $v_{i}$. The term $x_{i}(u)$ is the distance existing between the virtual source and the hydrophone along the $x$ axis

$$
x_{i}(u)=x_{0}-v_{i} u,
$$

where $v_{i}$ is considered positive for approaching sources and negative for receding sources. Expression (2) can be rewritten as

$$
u+\frac{\sqrt{\left(x_{0}-v_{i} u\right)^{2}+z_{i}^{2}}}{c}=t .
$$

In order to obtain the time of emission as a function of the time of reception, some hypotheses are necessary. The first one considers that the depth of the propagation channel can be neglected compared with its length. After calculation (given in Appendix B) and under our first hypothesis, expression (7) can be expressed by

$$
u+\frac{x_{0}-v_{i} u}{c}+\frac{z_{i}^{2}}{2 c\left(x_{0}-v_{i} u\right)}=t .
$$

The second hypothesis states that the distance covered by the moving source during one transmission can be neglected compared with the source-hydrophone separation. Some calculation (given in Appendix B) yields an approximation of the expression of the time of emission as a function of the time of reception:

$$
u=\frac{t-\tau_{i}}{1-v_{i}\left(\frac{1}{c}-\frac{z_{i}}{2 c x_{0}^{2}}\right)},
$$

where $\tau_{i}$ corresponds to the time-delay associated with the $i$ th path for a fixed source located at $x=x_{0}$,

$$
\tau_{i}=\frac{x_{0}}{c}+\frac{z_{i}^{2}}{2 c x_{0}} .
$$

Relation (9) means that the signal received for the $i$ th ray is a time-delayed and time compressed (or expanded) version of the transmitted one. Expression (10) illustrates what appears logical: if one computes an IR with a moving source and wants to compare it with the motionless case, it should be done with a source located in the middle of the motion.

\section{B. Signal received in multipath configurations}

In Eq. (6), it has been assumed that the speed of the source appears to be different for each ray. The projection of the source's speed on the sight line between the transmitter and the receiver is called $\vec{v}$. It is assumed that $\vec{v}$ can vary with time as the line defined by the source and the receiver changes. As shown in Fig. 1, the motion vector $\vec{v}$ is then projected on the path of the $i$ th ray with the declination angle $\theta_{i}$ which leads to

$$
v_{i}=\|\vec{v}\| \cos \left(\theta_{i}\right) .
$$

A solution of the wave equation for the sound field in an iso-speed ocean channel overlying a homogeneous fluid halfspace was developed and published over half a century ago in a classic paper by Pekeris. ${ }^{15}$ For Pekeris waveguides ${ }^{16}$ the departure angle of one path, $\theta_{i}$, and its angle of arrival differ in sign for one ray out of two but they have the same cosine. This basic property can be used to validate the assumption of using a Pekeris waveguide. We consider that the received signal is distorted by the combined effects of propagation and source motion (i.e., time-delayed, amplitude attenuated, and Doppler transformed). Using Eq. (9), and adding a change in amplitude to conserve energy yields the expression of the signal received at time $t$ for the $i$ th ray

$$
s_{i}(t)=a_{i} \eta_{i}^{1 / 2} e\left(\left(t-\tau_{i}\right) \cdot \eta_{i}\right),
$$

where $e(t)$ is the transmitted signal, $a_{i}$ represents the amplitude attenuation due to propagation losses, and $\eta_{i}$ is the scale factor due to the broadband Doppler effect satisfying

$$
\eta_{i}=\frac{1}{1-\|\vec{v}\| \cos \left(\theta_{i}\right)\left(\frac{1}{c}-\frac{z_{i}}{2 c \cdot x_{0}^{2}}\right)} .
$$

The received signal $s(t)$ is the sum of all the $s_{i}(t)$ received from each ray which leads to the following expression:

$$
s(t)=\sum_{i} s_{i}(t) .
$$

We made the hypothesis that the distance covered by the source during the transmission can be neglected compared with the source-receiver separation to obtain Eq. (8). From now on, we can consider the propagation time, $T_{i}(u)$, and the projection of the motion vector $\vec{v}$ along the path of the $i$ th ray, $v_{i}$, to be constant during one emission. By using the complete formulation of the signal received for each ray (12) and of the compression factor $\eta_{i}$ (13), Eq. (14) can be rewritten as

$$
s(t)=\sum_{i} a_{i} \eta_{i}^{1 / 2} e\left(\left(t-\tau_{i}\right) \eta_{i}\right) .
$$

This expression illustrates that the signal received from a moving source with a multipath propagation is a weighted sum of amplitude attenuated, time-delayed, and Dopplertransformed versions of the transmitted signal. The compression factor $\eta_{i}$ depends on the velocity of the source $\vec{v}$, on the angle of emission of ray $i$, and on the position of its corresponding virtual source, as shown in Eq. (13). 


\section{EFFECTS OF SOURCE MOTION}

In Sec. II, the received multipath signal has been characterized. The effects of source motion on the estimation of an IR with matched filtering are formulated and analyzed in this section.

\section{A. Effects of source motion on the matched-filter output}

Considering that the received signal is defined as the sum of amplitude-attenuated, time-delayed, and Dopplertransformed versions of the transmitted signal, the Dopplertransformation stands for a Doppler scaling which is not approximated as a simple frequency shifting. Assuming that the transmitted signal is known, the propagation time and the velocity associated with each ray can be estimated by cross correlating the received signal with a set of reference signals. The set of reference signals is composed of time-delayed and Doppler-transformed versions of the emitted signal for the range of time delays and speeds expected. ${ }^{14}$ For each reference signal, the cross correlation depends on the speed $v$ because of the $\eta$ dependency and is computed by

$$
R(\tau, v)=\int_{-\infty}^{\infty} s(t+\tau) \eta^{1 / 2} e^{T}(\eta t) d t
$$

where ${ }^{T}$ denotes the complex conjugation, $s(t)$ is the received signal, $e(t)$ is the transmission, and $\eta$ is the compression factor due to the Doppler effect. Using the expression of the received signal in a multipath environment (15) in Eq. (16) yields

$$
R(\tau, v)=\sum_{i} a_{i}\left(\eta \eta_{i}\right)^{1 / 2} \int_{-\infty}^{\infty} e\left(\eta_{i}\left(t+\tau-\tau_{i}\right)\right) e^{T}(\eta t) d t .
$$

Local maxima of this correlation function are reached for each ray. For the $i$ th ray, the maximum is reached when the reference and the propagated signal are exactly aligned in time delay and Doppler. It is assumed that the smallest time difference between two consecutive arrivals is larger than the inverse of the time-bandwidth product of the transmitted signal so rays are well separated for the motionless case, and each peak of the correlation can be detected. The interferences that could occur between local maxima are studied in

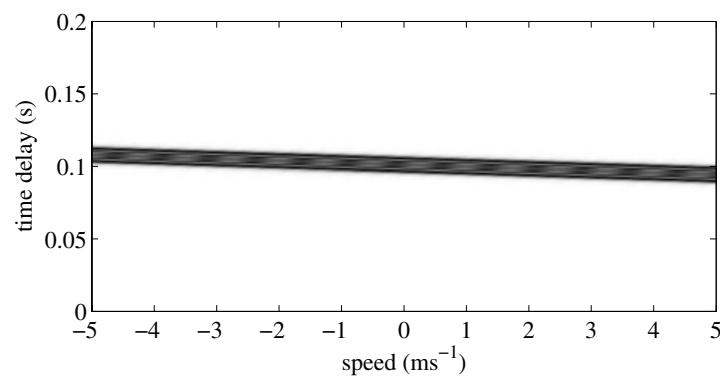

FIG. 2. Representation of a LFM in the narrowband ambiguity plane without multipath with a central frequency of $1300 \mathrm{~Hz}$, a bandwidth of $2000 \mathrm{~Hz}$, and a time duration of $4 \mathrm{~s}$. The simulated speed is $2.5 \mathrm{~ms}^{-1}$ and the time delay is $0.1 \mathrm{~s}$.
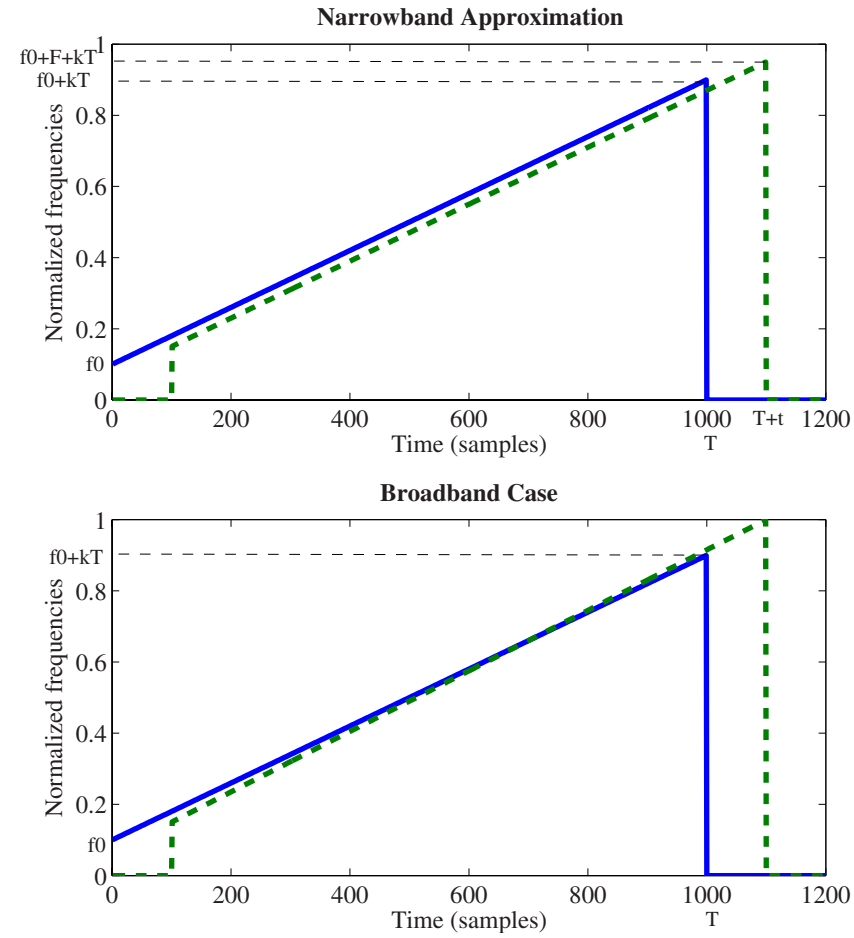

FIG. 3. (Color online) Time frequency ideal representation illustrating the mismatch existing between the transmitted LFM signal (solid line) and the Doppler-transformed signal (dashed line) for the narrowband approximation and the broadband case. $f 0$ is the beginning frequency of the LFM.

Ref. 14 and are not the purpose of this paper. The time of propagation and the apparent speed of the $i$ th ray can be estimated once a local maximum is detected.

\section{B. Effects of source motion on the ambiguity plane}

The wideband ambiguity plane introduced here is the squared magnitude of the result of the correlation equation (17). The propagation time and the velocity associated with the received signal are estimated by cross correlating the received signal with a set of reference signals. The set of reference signals is composed of time-delayed and Dopplertransformed versions of the transmitted signal which is assumed to be known.

For geoacoustic inversion applications, the emitted signals are wideband signals, and the motion effect cannot be approximated by a frequency shifting. It is well known that the representation of a linear frequency modulation (LFM) signal in the narrowband ambiguity plane is ambiguous, as shown in Fig. 2. A LFM frequency shifted by the narrowband approximation of the Doppler effect is really close to one another which is just time-delayed, as illustrated in Fig. 3 . Figure 3 shows the ideal time frequency representation of a LFM signal compared with the Doppler-transformed versions of the signal under narrowband and broadband approximations of the Doppler effect. That is why there is an ambiguity and one cannot find accurately the right coordinate of the maximum in the ambiguity plane. There is no absolute maximum in the narrowband ambiguity plane, and possible solutions are represented by all the points of the 


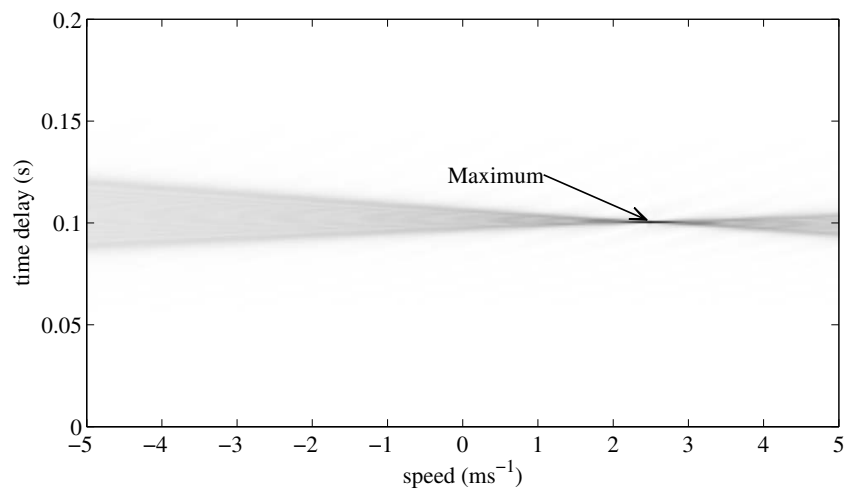

FIG. 4. Representation in the wideband ambiguity plane of a LFM without multipath with a central frequency of $1300 \mathrm{~Hz}$, a bandwidth of $2000 \mathrm{~Hz}$, and a time duration of $4 \mathrm{~s}$. The simulated speed is $2.5 \mathrm{~ms}^{-1}$ and the time delay is $0.1 \mathrm{~s}$.

straight line containing the energy. Hence neither the propagation time nor the source speed can be estimated accurately when a broadband LFM is transmitted.

As shown in Fig. 4, the representation of a time-delayed and Doppler-transformed LFM provides a finite resolution in the $(\tau, v)$ domain but remains ambiguous in the wideband ambiguity plane, although there is one absolute maximum that can be detected far more accurately than in the narrowband ambiguity function. That is why the wideband ambiguity plane is well adapted to geoacoustic inversion applications. The maximum is reached when the parameters of the reference signal match exactly with the parameters of the estimate. The amplitude of the correlation stays high for time delays close to the simulated one, and the correlation broadens farther from the simulated speed.

For multipath propagation, paths have different apparent speeds and different time delays, as illustrated in Fig. 5. Each path is seen as a sweep-like shape which broadens with the distance between the reference and the simulated speed. Figure 5 illustrates the wideband ambiguity plane for a simulated multipath propagation centered on the six first arrivals.

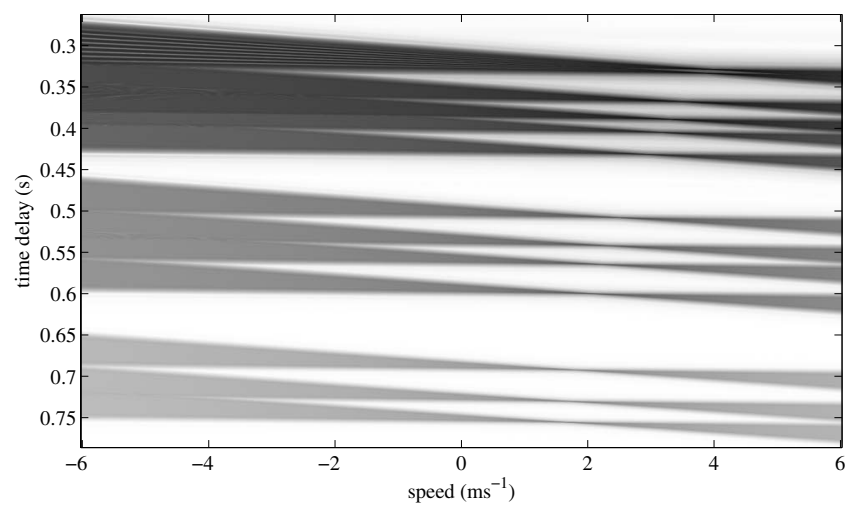

FIG. 5. Ambiguity plane of a simulated multipath propagation. The relative speed simulated is almost 8 knots and the source-receiver separation is $500 \mathrm{~m}$. The signal transmitted is a LFM with a central frequency of $1300 \mathrm{~Hz}$, a bandwidth of $2000 \mathrm{~Hz}$, and a time duration of $4 \mathrm{~s}$. The propagation channel is $165 \mathrm{~m}$ deep with a constant sound speed of $1500 \mathrm{~ms}^{-1}$. The bottom is a half space with a sound speed of $1800 \mathrm{~ms}^{-1}$ and a density of $1800 \mathrm{~kg} \mathrm{~m}^{-3}$.

\section{The LFM case}

LFMs are classically used for geoacoustic inversions and tomography because their large time-bandwidth product provides a good resolution and because of electro-acoustic transduction technological constraints. From now on, the transmitted signal is assumed to be a LFM signal with known parameters

$$
e(t)=\frac{\operatorname{rect}\left(\frac{t}{T}\right)}{\sqrt{(T)}} \exp \left(j 2 \pi\left(f_{c} t+\frac{k}{2} t^{2}\right)\right),
$$

where $f_{c}$ is the central frequency of the sweep, $k$ is the chirp rate or sweep rate, $T$ is the duration of the signal, and the rect function is defined by

$$
\operatorname{rect}(t)= \begin{cases}1 & \text { if }|t| \leq \frac{1}{2} \\ 0 & \text { otherwise }\end{cases}
$$

Some algebraic manipulations ${ }^{14,17,18}$ detailed in Appendix C with Eqs. (17) and (18) lead to the analytic expression of the cross correlation defined previously if $v$ is different from $v_{i}$,

$$
\begin{aligned}
& R(\tau, v)=\sum_{i} \frac{C_{i} D_{i} E_{i}}{2 \sqrt{\left|\beta_{i}\right|}} \int_{X_{i}}^{Y_{i}} \exp \left(\gamma \frac{\pi}{2} j t^{2}\right) d t, \\
& \text { where } \quad C_{i}=\frac{\eta_{i}^{1 / 2} \eta^{1 / 2} a_{i}}{T} \exp \left(j \pi\left(f_{c} \Delta \tau_{i}\left(\eta+\eta_{i}\right)\right)\right) \text {, } \\
& D_{i}=\exp \left(\pi \frac{k \Delta \tau_{i}^{2}}{4} j\left(\eta_{i}^{2}-\eta^{2}\right)\right) \\
& E_{i}=\exp \left(-\gamma 2 j \pi\left(\frac{\alpha_{i}}{2 \sqrt{\left|\beta_{i}\right|}}\right)^{2}\right) \\
& \beta_{i}=\frac{k}{2}\left(\eta_{i}^{2}-\eta^{2}\right) \\
& \alpha_{i}=f_{c}\left(\eta_{i}-\eta\right)+k \Delta \tau_{i}\left(\eta^{2}-\eta_{i}^{2}\right), \\
& \Delta \tau_{i}=\tau-\tau_{i}, \\
& X_{i}=\frac{\gamma \alpha_{i}}{\sqrt{\left|\beta_{i}\right|}}+2 t_{1} \sqrt{\left|\beta_{i}\right|} \\
& Y_{i}=\frac{\gamma \alpha_{i}}{\sqrt{\left|\beta_{i}\right|}}+2 t_{2} \sqrt{\left|\beta_{i}\right|} \\
& \gamma=\operatorname{sgn}\left(k\left(\eta_{i}-\eta\right)\right) .
\end{aligned}
$$

The bounds of integration of Eq. (20) depend on $t_{1}$ and $t_{2}$ which are given in Appendix D. Finally, the result of Eq. (20) can be expressed and simplified with a complex form of the Fresnel integrals if $v$ is different from $v_{i}$,

$$
R(\tau, v)=\sum_{i} \frac{C_{i} D_{i} E_{i}}{2 \sqrt{\left|\beta_{i}\right|}}\left(F\left(Y_{i}\right)-F\left(X_{i}\right)\right),
$$




$$
\begin{aligned}
& F(u)=C(u)+j \gamma S(u), \\
& C(u)=\int_{0}^{u} \cos \left(\frac{u t^{2}}{2}\right) d t, \\
& S(u)=\int_{0}^{u} \sin \left(\frac{\pi t^{2}}{2}\right) d t .
\end{aligned}
$$

When the Doppler transformation of the reference signal matches exactly the Doppler transformation of the $i$ th path, expression (21) is no longer valid, and the $i$ th term of the sum, $r_{i}\left(\tau, v_{i}\right)$, becomes

$$
\begin{aligned}
& r_{i}\left(\tau, v_{i}\right)=C_{i}\left(\left|\Delta \tau_{i}\right|-\frac{T}{\eta_{i}}\right) \frac{\sin \left(\xi_{i}\right)}{\xi_{i}}, \\
& \xi_{i}=\pi k \Delta \tau_{i}\left(\eta_{i}\left|\Delta \tau_{i}\right|-T\right) .
\end{aligned}
$$

When the reference signal matches exactly the $i$ th path, expression (25) reaches its maximum as expected and equals $a_{i}$ meaning that the amplitude associated with each ray can be recovered. It is worthy noting that Eq. (25) is a sine cardinal multiplied by a constant that can be compared with the classical LFM ambiguity function. According to the asymptotic evaluation of Harris and $\mathrm{Kramer}^{19}$ and Kramer, ${ }^{17}$ the Doppler tolerance, i.e., half-power contour, is given by

$$
V_{-3 \mathrm{~dB}}= \pm \frac{2610}{T W} \text { knots, }
$$

where $T$ is the duration of the LFM and $W$ the signal bandwidth. As an example, we consider a large $T W$-product and wideband LFM signal with known parameters:

$$
f_{c}=1300 \mathrm{~Hz}, \quad W=2000 \mathrm{~Hz}, \quad T=4 \mathrm{~s},
$$

where $f_{c}$ is the central frequency of the LFM. From Eq. (26) the Doppler tolerance of this signal in the wideband ambiguity plane is $V_{-3 \mathrm{~dB}}= \pm 0.32$ knots, whereas its classical Doppler tolerance in the narrowband ambiguity plane would be

$$
V_{-3 \mathrm{~dB}}= \pm \frac{450 \mathrm{~W}}{f_{c}}= \pm 692.3 \text { knots. }
$$

This example confirms the results obtained with our wave propagation modeling, the wideband ambiguity function is well adapted for the study of Doppler scenarios in geoacoustic inversion applications, and the narrowband approximation is not valid. For the narrowband case, the correlation receiver has a reference set of signals composed of time-delayed and carrier-frequency-shifted versions of the transmitted signal. As can be seen in Fig. 3, a LFM frequency-shifted by the effects of motion is not very different from one another which is only time-delayed; that is why LFM signals have a poor Doppler tolerance and are ambiguous in the narrowband ambiguity plane. The inclusion of the motion effect for broadband signals, i.e., time compression (or expansion), in the computation of the correlation receiver clearly enhances the Doppler tolerance of wideband signals so that a LFM is no longer ambiguous in the wideband am-
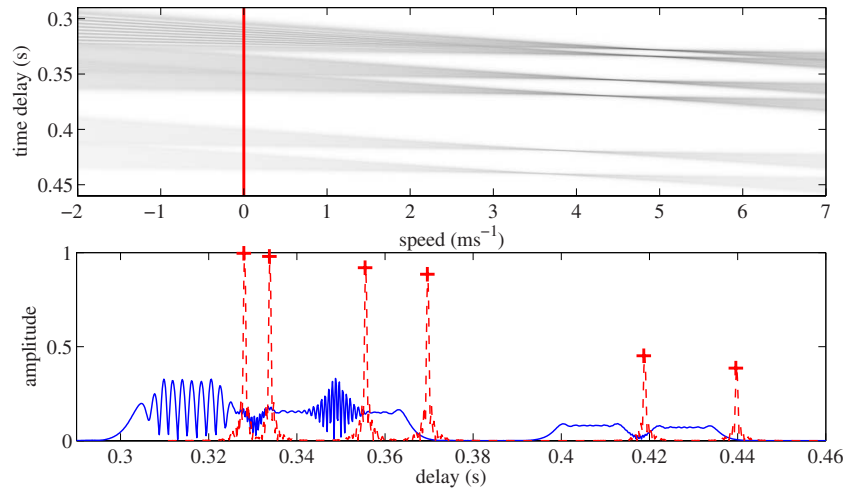

FIG. 6. (Color online) Estimation of the IR of a propagation channel computed by keeping a column at zero speed in the ambiguity plane represented by solid lines. The dotted line represents the IR estimated with a motionless source. The relative speed simulated is almost 10 knots and the sourcereceiver separation is $500 \mathrm{~m}$.

biguity plane. Different Doppler removal techniques derived from the wideband ambiguity plane are introduced and studied in Sec. IV.

\section{DOPPLER REMOVAL TECHNIQUES}

A computational method has been presented in Sec. III to study the multipath propagation when a LFM is transmitted. The wideband ambiguity plane was introduced as an adapted representation of the multipath propagation for geoacoustic applications whenever the source is moving or not.

\section{A. The motionless source hypothesis}

A well known method of estimating the IR of a propagation channel is to compute the cross correlation between the time-delayed transmitted signal and the received signal as

$$
\operatorname{IR}(\tau)=\sum_{i} a_{i} \eta_{i} \int_{-\infty}^{\infty} e\left(\eta_{i}\left(t+\tau-\tau_{i}\right)\right) e^{T}(t) d t .
$$

This is equivalent to computing expression (17) with a zero speed, meaning $\eta=1$, for all references. The result of Eq. (29) is a sub-part of the broadband ambiguity plane and can be obtained by keeping the column at zero speed in this plane. From Eq. (29) it can be seen that the motion effects are not considered and the computed IR will be biased. Both the estimation of the time of propagation $\left(\tau_{i}\right)$ and the amplitude of the $i$ th ray $\left(a_{i}\right)$ will be incorrect. An example of the zero speed correlation is illustrated with the solid line in Fig. 6 . The parameters used for this simulation are the same as the one used for the simulation presented in Fig. 5 except for the source speed. The first two paths are not resolved because the transmitted signal is transformed by the Doppler effect which is not taken into account during the processing. The amplitude of each peak is lowered and the time delays are not correctly estimated. The exact values of amplitude and time delay of the $i$ th path are located at the maximum value of the shape associated with the $i$ th path on the wideband ambiguity plane. The effects of Doppler transformations on wideband LFM signals have been verified for a large set of simulated data, confirming the necessity of adding a 


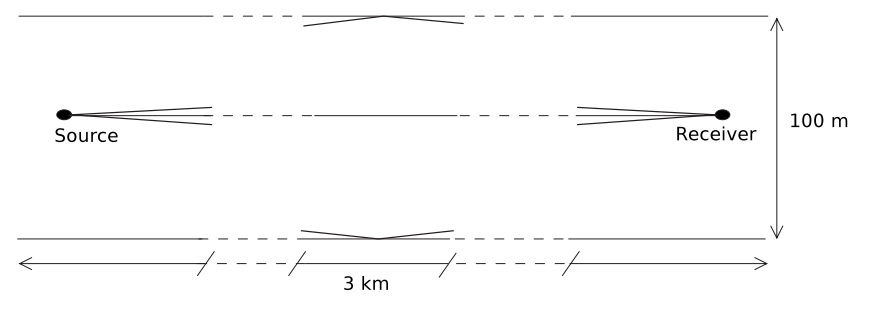

FIG. 7. Schematic illustration of the hypothesis necessary to obtain relation (30). Here the source is 30 times farther than the channel depth and only the first three paths are represented for simplicity.

speed parameter to the matched filtering processing so the motion of the source can be estimated and then compensated.

\section{B. The uniform speed compensation}

The cross correlation between the time-delayed transmitted signal and the received signal has been shown to have poor performances in estimating the IR when the motion is not taken into account. We propose here a new method to compensate the motion in the estimation of an IR of the propagation channel. This aims at reconstructing a motionless IR from an acoustic observation with a moving source in order to enable the use of classical acoustic inversion processes. The declination angle of the direct path is very low, and expression (11) shows that the apparent speed of this path will be the projection of the motion vector along the receiver-source line. If the amplitude of the direct path is considered higher than any other, then the amplitude of its correlation with the reference signals is also higher than any other. The speed of the source $v$ is estimated as the coordinates of the absolute maximum in the ambiguity plane. If the source is far away from the receiver, as illustrated in Fig. 7 , the received paths will have low declination angles, meaning an apparent speed close to $v$ and Eq. (11) becomes

$$
v_{i}=\|\vec{v}\| \text {. }
$$

The uniform speed compensation is defined as keeping the column at constant speed $v$ in the wideband ambiguity plane and is presented in Fig. 8.

We developed a software simulating all the propagation process with ray theory for signals transmitted from a moving source in order to test our uniform motion compensation method. The simulation is presented in Fig. 8 and was computed with a source moving at a constant speed of $5 \mathrm{~ms}^{-1}$, at a depth of $24 \mathrm{~m}, 4 \mathrm{~km}$ from the receiver at a depth of $90 \mathrm{~m}$ on a $165 \mathrm{~m}$ deep channel with a constant sound speed of $1500 \mathrm{~ms}^{-1}$. The signal transmitted is a LFM with a central frequency of $1300 \mathrm{~Hz}$, a bandwidth of $2000 \mathrm{~Hz}$, and a duration of 4 s. In Fig. 8, the star shows the absolute maximum detected in the wideband ambiguity plane, giving an estimated speed of $5 \pm 0.16 \mathrm{~ms}^{-1}$ as expected. In this case, the source is far away from the receiver at a distance much greater than the depth of the propagation channel so the conditions necessary to apply uniform speed compensation are met. The compensation of the motion on the estimated IR is made by keeping the column at the estimated speed which is represented with solid line on the top of Fig. 8. The panel on the bottom of Fig. 8 represents estimates of the IR where the
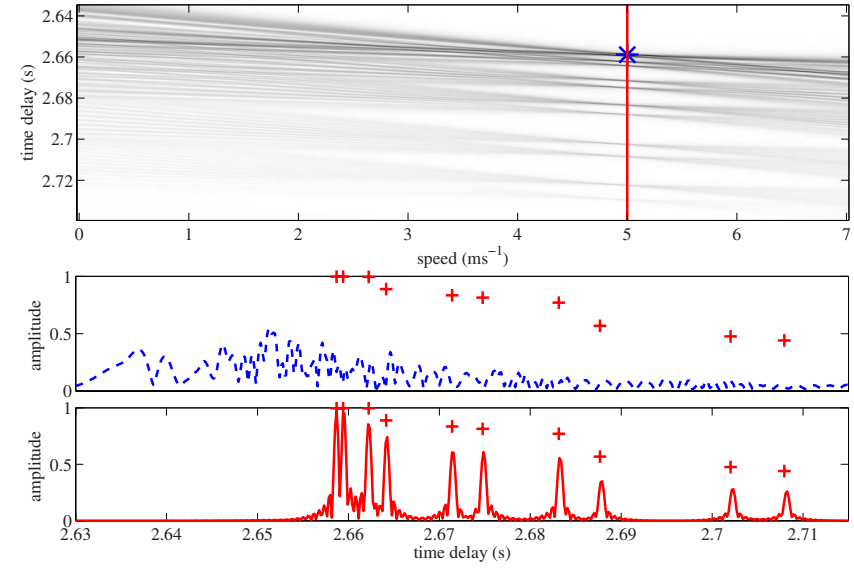

FIG. 8. (Color online) The top panel shows the ambiguity plane for a $4000 \mathrm{~m}$ multipath propagation. The star represents the detected absolute maximum. The bottom panel shows the IR estimated with zero speed compensation in dashed lines (on the top); the IR estimated with uniform speed compensation is represented with solid line (on the bottom) and the crosses stand for the ideal simulated IR.

dashed line stands for the IR estimated with zero speed compensation (no speed compensation). The solid line stands for the estimation of the motion compensated IR while the crosses represent the simulated IR. This compensation method is effective and improves the estimated IR. Both amplitudes, time delays, and peak detections have been improved. It is not possible to distinguish any path without compensation while they are clearly detectable with compensation. The general shape of the IR is well recovered even if amplitudes are not the theoretical ones.

The estimated amplitudes of the motion compensated IR are biased because the hypothesis made in relation (30) is not valid for all rays, and the column at constant speed $v$ does not cut each chirp-like shape around its maximum in the ambiguity plane. The interferences existing between paths are clearly illustrated on the broadband ambiguity plane of Fig. 8. As expected, the Doppler effect lowers amplitudes, shifts time delays, and leads to the appearance of interferences between peaks which are not detectable if the motion is not compensated. The time delay shifting is clear on the estimated IR represented in Fig. 8 and can be explained by the sweep-like shape of each path on the ambiguity plane.

Figure 9 illustrates another example of uniform speed compensation where the source is moving at a speed of $4 \mathrm{~ms}^{-1}$ at a depth of $32 \mathrm{~m}$ and a distance of $500 \mathrm{~m}$ from the receiver. The recorder is at a depth of $90 \mathrm{~m}$ and the propagation channel is the same as before. The star represented on the wideband ambiguity plane of Fig. 9 shows the absolute maximum detected in the wideband ambiguity, giving an estimated speed of $3.99 \pm 0.16 \mathrm{~ms}^{-1}$ which is valid. For this simulation, the depth of the channel cannot be neglected compared with the source-hydrophone separation so the conditions required to apply the uniform speed compensation are not met. We consider that the uniform speed compensation can be applied when the source-hydrophone separation is at least ten times larger than the channel depth. The motion compensation for the IR estimate is made by keeping the column at the estimated speed which is represented with 

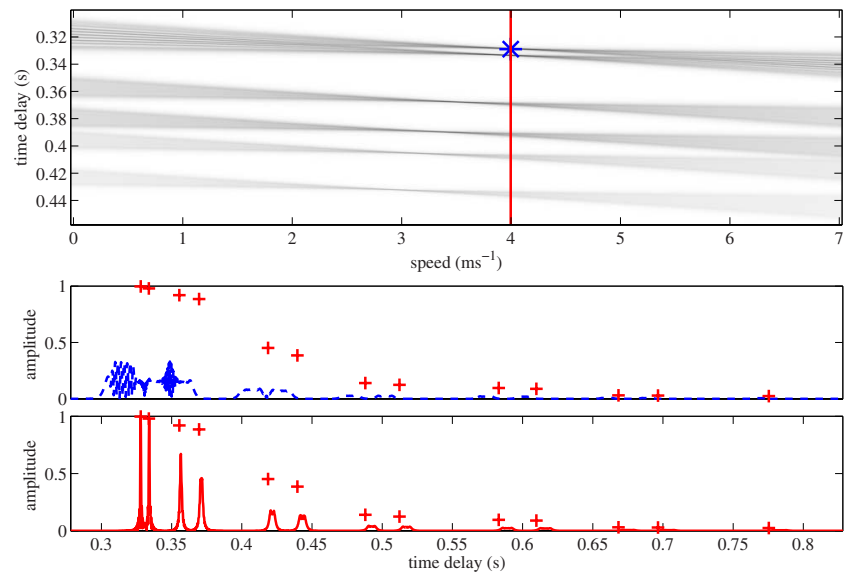

FIG. 9. (Color online) The top panel shows the ambiguity plane for $500 \mathrm{~m}$ multipath propagation. The star represents the detected absolute maximum. The panel on the bottom shows the IR estimated with zero speed compensation in dashed lines (on the top); the IR estimated with uniform speed compensation is represented with solid line (on the bottom) and the crosses stand for the ideal simulated IR.

solid line in Fig. 9. Contrary to the previous simulation, it can be seen that the line along which the compensation is made on the ambiguity plane is no longer cutting each sweep-like shape around its maximum. This means that the apparent speed, amplitude, and time delay of each ray will not be estimated properly. In Fig. 9, it is worthy noting that the distance between local maxima and the line along which the compensation is made increases with the time delay, which explains the degradation of estimations for the last rays. These drawbacks can also be seen on the estimation of the motion compensated IR which is represented by a solid line in the bottom of Fig. 9 and can be compared with the dashed line representing the zero speed compensation and the crosses representing the simulated IR. The first two rays of the motion compensated IR are well estimated and their time-delays and amplitudes are well recovered. However, the estimation error of the motion compensated IR increases with the time-delays.

The uniform speed compensation is a good way to compensate the motion of a source while estimating an IR. Though the hypothesis is necessary, its validity cannot always be verified. A compensation method that considers the speed of each ray individually should lead to a more general result and is one of our short term prospects. ${ }^{20}$

\section{APPLICATION TO REAL DATA}

In this section, the previously described methods are tested on a dedicated real data set recorded in a shallow water environment.

\section{A. Experiment description}

The BASE'07 experiment was jointly conducted by the NATO Undersea Research Center, the Forschungsanstalt der Bundeswehr für Wasserschall und Geophysik, the Applied Research Laboratory, and the Service Hydrographique et Ocanographique de la Marine (SHOM). The main objective of the experiment is to investigate broadband adaptive sonar techniques in the shallow water. Two additional days of mea-

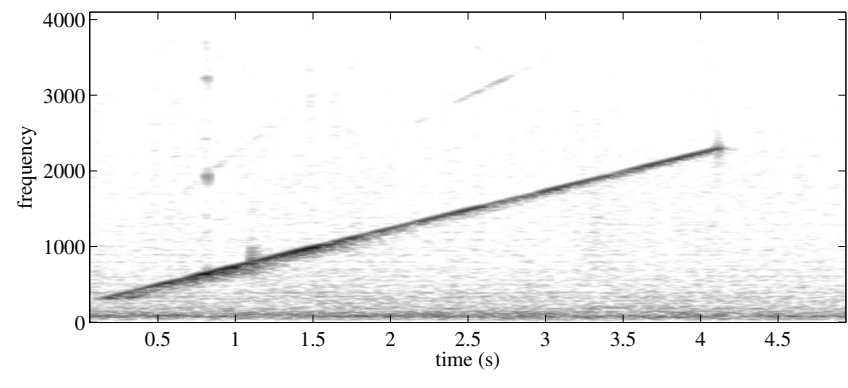

FIG. 10. Spectrogram of the LFM signal transmitted by the towed source. The effects of the multipath underwater propagation such as the apparition of time-delayed echoes can be seen on this time frequency representation.

surement were also conducted by SHOM for geoacoustic inversion. Some results can be seen in Refs. 21-23. The first day was dedicated to a general survey and the second day was dedicated to motion effect compensation. The real data of this second day are used in the following. The campaign took place on the Malta Plateau in shallow water $(130 \mathrm{~m}$ depth). Underwater LFM, as illustrated in Fig. 10, was transmitted by a source moving rectilinearly at constant speed from 2 to 12 knots and different depths. The transmitted LFM has a bandwidth of $2000 \mathrm{~Hz}$, a central frequency of $1300 \mathrm{~Hz}$, and a duration of $4 \mathrm{~s}$, and the effects of the multipath propagation can be seen in Fig. 10. The transmitted signal's bandwidth is very large compared with the signal's central frequency, which ensures a large propagation distance and good auto-correlation properties for geoacoustic inversion. However, the transmitted signals are Doppler sensitive. The source-receiver separation varied from 500 to $25000 \mathrm{~m}$, and the transmitted signals were recorded by an array of six hydrophones located at different depths (from 9 to $94 \mathrm{~m}$ ). As shown in Fig. 11, the array of hydrophones had its own global positioning system (GPS) and clock for localization and was not anchored so it can move freely with currents and avoid additional flow noise. The boat had a GPS which was used to derive the position of the towed source. Both position and speed of the source and the hydrophone array are known at any moment so the results can be compared and analyzed.

\section{B. Results}

The wideband ambiguity plane has been studied for more than 100 different scenarios on each of the six hydrophones of the array. The motion existing between the source and the receivers was clearly seen on all the wideband ambiguity planes. The uniform speed compensation method was then automatically carried out to estimate the source-receiver relative speed and the motionless IR. All the broadband ambiguity planes analyzed from real data give realistic results. They are close to the simulated data and the chirp-like shapes, representing that each path of the acoustic waves is easily seen. The apparent speed of the first path is estimated in the ambiguity plane by the detection of the absolute maximum. It corresponds to the projection of the speed vector on the sight line existing between the source and the hydrophones multiplied by the cosine of the declination angle. The accuracy of the speed estimate using Eq. (26) is $0.16 \mathrm{~ms}^{-1}$ or 0.32 knots. An example of the wideband ambiguity plane 

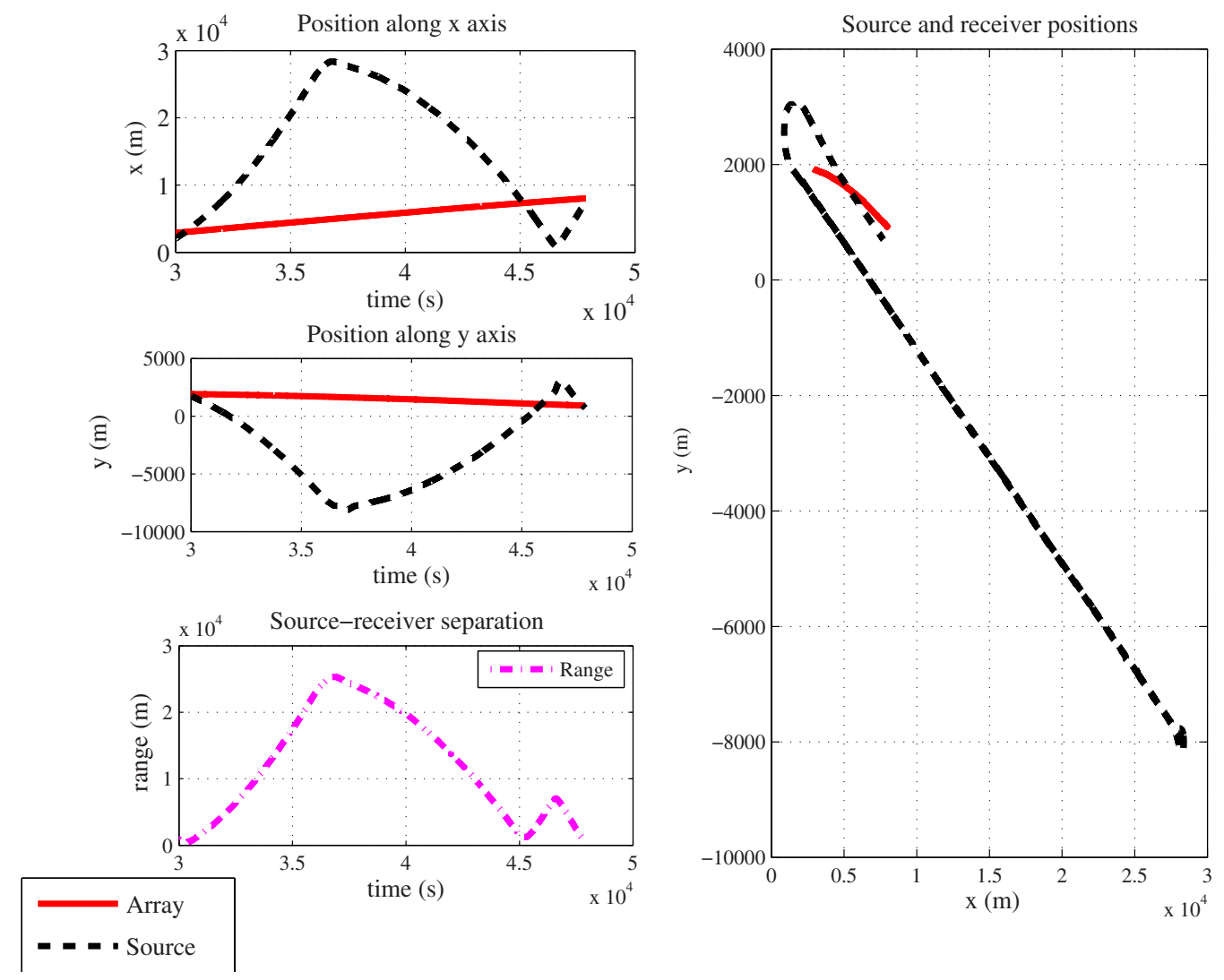

FIG. 11. (Color online) Positions of the towed source and of the hydrophones along time and evolution of source-receiver separation along time.

with its associated motion-compensated IR is presented in Fig. 12. The scenario presented here was recorded with a projected speed of $11 \mathrm{knots}$, a source at $24 \mathrm{~m}$ depth, and a $4015 \mathrm{~m}$ propagation channel. The speed estimated by the automatic process is 10.8 knots which stays within the bounds of accuracy. The ambiguity plane illustrated on the top of Fig. 12 is close to that obtained during simulations. The IR estimated with the uniform speed compensation method is shown as a solid line on the bottom panel of Fig. 12. The amplitude of each peak seems to be corrected, and the time delays are shifted compared with those of the IR estimated with classical matched filtering represented with a dashed line. It is worthy noting that the source perpetually moves so the propagation channel is different for each emission. This shows that it is not possible to improve the IR by computing means as is usually done with real data and motionless sources. A simulated IR obtained from a Pekeris waveguide with a flat bottom of sandy mud having a sound speed of $1550 \mathrm{~ms}^{-1}$ and a density of $1700 \mathrm{~kg} \mathrm{~m}^{-3}$, which is close to the data recorded in situ, is represented with crosses on the bottom panel of Fig. 12. The propagation speed of the simulated canal is $1500 \mathrm{~ms}^{-1}$ and its depth is $125 \mathrm{~m}$. Even if the simulated IR is not the real one, this provides a reference to compare the IR estimated with classical matched filtering and the motion-compensated IR. It can be seen that the uniform speed compensation both corrects the general shape of the IR and shifts the time delays which was the case for simulations. Finally the motion compensated IR is closer to the expected IR than the IR computed with zero speed com-
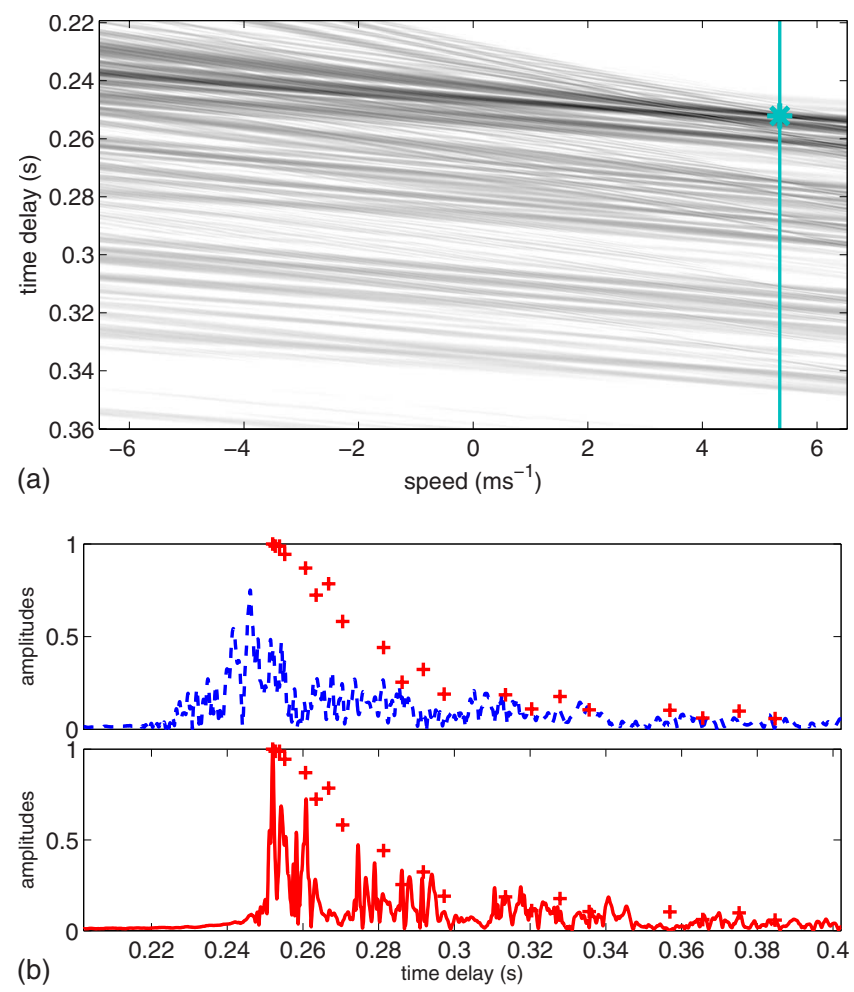

FIG. 12. (Color online) The top panel shows the wideband ambiguity plane for real data from BASE'07 campaign. The two lower panels represent the IR estimated with classical matched filtering (dashed line) and the motioncompensated IR (solid line). The crosses represent the IR simulated with a Pekeris waveguide having parameters close to the data recorded in situ. 
TABLE I. The mean and standard deviation (std) of the difference between the estimated speed and the projection of the real speed normalized by the projection of the real speed expressed in $\mathrm{m} / \mathrm{s}$. 70 transmissions have been studied with a range source-receiver varying from 1500 to $6000 \mathrm{~m}$ and a source's speed varying from -4 to $6 \mathrm{~ms}^{-1}$.

\begin{tabular}{ccccccc}
\hline \hline & $\mathrm{H} 1$ & $\mathrm{H} 2$ & $\mathrm{H} 3$ & $\mathrm{H} 4$ & $\mathrm{H} 5$ & $\mathrm{H} 6$ \\
\hline Mean & 0.11 & 0.09 & 0.15 & 0.20 & 0.03 & 0.12 \\
std & 0.36 & 0.33 & 0.47 & 0.45 & 0.21 & 0.34 \\
\hline \hline
\end{tabular}

pensation, showing the improvements of our compensation method on real data.

Most of the estimated speeds were correct and quite accurate, leading to good compensation of the motion, but some were poor. Results obtained on the estimated speeds from 70 transmissions are presented and analyzed for each hydrophone in Table I. In most cases, poor speed estimates occurred when the absolute maximum of the ambiguity plane corresponded to constructive interference between two paths arriving almost simultaneously or because the distance between the source and the receiver exceeds $15000 \mathrm{~m}$. Finally, results are consistent from one hydrophone to another even if the signal to noise ratio (SNR) varies.

As illustrated in Fig. 13, the motion-compensated IR obtained from several hydrophones at different depths has been analyzed and compared with classical matched filtering IR. The IRs estimated from the data recorded on hydrophone number one (H1) match well with the physics of the propagation channel. This hydrophone is located close to the sea surface in the mixed layer where the sound speed is almost constant. The upper left of Fig. 13 clearly shows a family of rays arriving first with low amplitude which were trapped close to the sea level and were reflected at the sea surface. For this application to real data, the motion compensation improves the shape of the IR and allows recovery of the
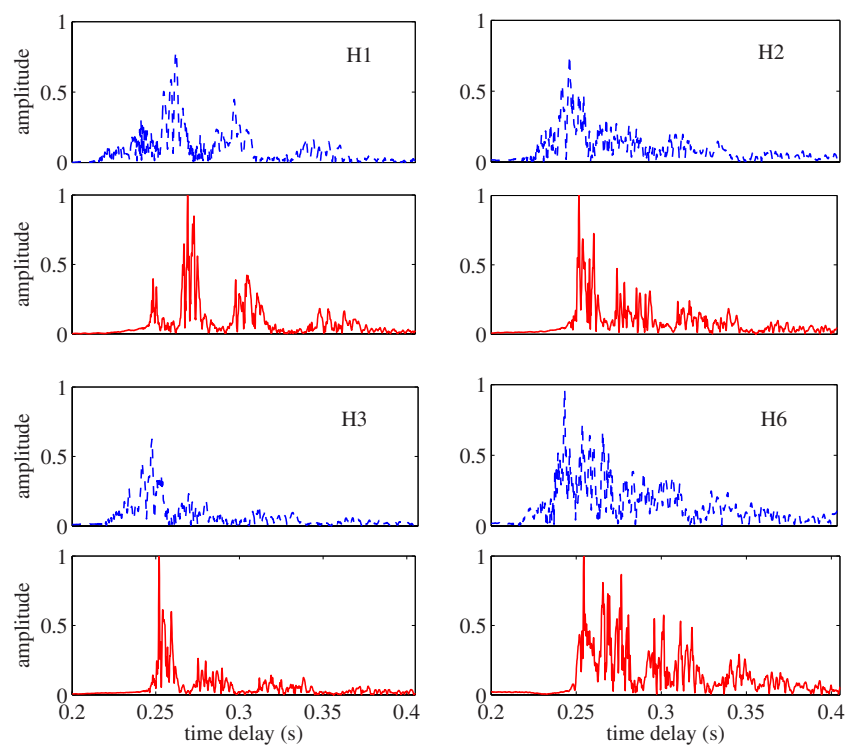

FIG. 13. (Color online) Time series of the IR estimated from four different hydrophones at different depths. The dashed lines represent the IR estimated with classical matched filtering and the solid lines stand for the motioncompensated IR. The depths of the hydrophones are $\mathrm{H} 1$ at $-9 \mathrm{~m}, \mathrm{H} 2$ at $-82.5 \mathrm{~m}, \mathrm{H} 3$ at $-93.5 \mathrm{~m}$, and $\mathrm{H6}$ at $-51 \mathrm{~m}$. arrival time of each group of rays. Hydrophones number two and three $(\mathrm{H} 2$ and $\mathrm{H} 3)$ are located around sound speed minimum where the records have a high SNR. From H2 and H3 in Fig. 13, it can be seen that the motion compensated IRs have been corrected in time delays and amplitudes and present thinner shapes for each group of rays. Finally, hydrophone number six (H6) is located in the middle of the water column and its records have a low SNR, which leads to poor estimates of the IR for both the classical matched filtering and motion compensation methods.

\section{CONCLUSIONS}

The effects of motion while estimating the IR for shallow water environments with signals having low central frequencies and high bandwidth must be taken into account. In this case, Doppler effects that cannot be modeled by a carrier frequency shift usually used to represent the narrowband cases, and wideband Doppler effects should be considered instead, modeled as a compression or expansion in time. The wideband ambiguity plane is presented here as a convenient way of representing multipath environments in a transmitterreceiver motion scenario. The uniform compensation method for motion effect compensation is proposed in the wideband ambiguity plane in order to reduce the distortions due to motion when the transmitted signal is known. This compensation method was tested on real set of data from BASE'07 campaign (SHOM, South of Sicilia, 2007) leading to realistic results.

\section{ACKNOWLEDGMENT}

This work was supported by Délégation Générale pour l'Armement (DGA) under SHOM research Grant No. N07CR0001.

\section{APPENDIX A: EXPRESSIONS OF THE VIRTUAL SOURCE HEIGHT FOR A PROPAGATION CHANNEL WITH A CONSTANT SPEED}

This appendix gives the expressions of $z_{i}$ introduced in formula (5) for all rays considering that the propagation speed is constant. There are four possible expressions for $z_{i}$ depending whether the number of reflections is even or odd, and on the first reflection. The first family of rays is the $2 p$ +1 rays which has an odd number of reflections and begins with a reflection at the surface of the sea. The expression for the height of the corresponding virtual source is

$$
z_{i}^{2 p+1}=2(p+1) z_{s}-2 p z_{b}-z_{h},
$$

where $z_{s}, z_{b}$, and $z_{h}$ are the heights of the surface, of the bottom, and of the hydrophone compared to the position of the real source, respectively. $p$ is an integer and $2 p+1$ is the number of reflections. The second family of ray is $2 p$ rays which has an even number of reflections and begins with a reflection at the surface of the sea. The expression for the height of the corresponding virtual source becomes

$$
z_{i}^{2 p}=2 p z_{b}-2 p z_{s}-z_{h},
$$

where $p$ is an integer and $2 p$ is the number of reflections. The third family of rays is the $-(2 p+1)$ rays which has an odd 
number of reflections and begins with a reflection at the bottom of the propagation channel. The height of the corresponding virtual source is given by

$$
z_{i}^{-(2 p+1)}=2(p+1) z_{b}-2 p z_{s}-z_{h},
$$

where $p$ is an integer and $2 p+1$ is the number of reflections. Finally, the last family of rays, the $-2 p$ rays, is made of rays having an even number of reflections beginning with a reflection at the bottom of the sea. The height of the corresponding virtual source follows,

$$
z_{i}^{-2 p}=2 p z_{s}-2 p z_{f}-z_{h},
$$

where $p$ is an integer and $2 p$ is the number of reflections.

\section{APPENDIX B: CHARACTERIZATION OF THE MULTIPATH RECEIVED SIGNAL}

This appendix aims at explaining the calculation necessary to obtain expressions (8) and (9). The notations used in this part are the same as in Sec. II. The hypothesis that channel depth can be neglected compared with its length can be summarized as

$$
\left(x_{0}-v_{i} u\right)^{2} \gg z_{i}^{2} \text {. }
$$

This hypothesis is used to approximate $L_{i}(u)$ defined in Eq. (5) and get a linear expression of $u$ as a function of $t$. Expression (5) can be reformulated as

$$
L_{i}(u)=\left(x_{0}-v_{i} u\right) \sqrt{1+\frac{z_{i}^{2}}{2\left(x_{0}-v_{i} u\right)^{2}}} .
$$

Using hypothesis (B1) in Eq. (B2) yields

$$
L_{i}(u) \approx x_{0}-v_{i} u+\frac{z_{i}^{2}}{x_{0}-v_{i} u} .
$$

The expression of $L_{i}(u)$ obtained in Eq. (B3) is then injected in Eq. (7) which leads directly to expressions (8) and (B4),

$$
u+\frac{x_{0}-v_{i} u}{c}+\frac{z_{i}^{2}}{2 c\left(x_{0}-v_{i} u\right)}=t
$$

The first hypothesis (B1) allows expressing $u$ as a function of $t$, but this expression is not yet linear. The second hypothesis made in Sec. II assumes that the distance the source moves during one transmission can be neglected compared with the source-hydrophone separation, which can be summarized as

$$
x_{0} \gg v_{i} u \text {. }
$$

Hypothesis (B5) approximates the part of Eq. (B4) containing the inverse of the time of emission $u$ as

$$
\frac{z_{i}^{2}}{2 c\left(x_{0}-v_{i} u\right)} \approx \frac{z_{i}^{2}}{2 c x_{0}}+\frac{z_{i}^{2}}{2 c x_{0}^{2}} .
$$

Some manipulations with Eqs. (8) and (B6) finally lead to the expression of $u$ as a linear function of $t$ :

$$
u=\frac{t-\left(\frac{x_{0}}{c}+\frac{z_{i}^{2}}{2 c x_{0}}\right)}{1-v_{i}\left(\frac{1}{c}-\frac{z_{i}}{2 c x_{0}^{2}}\right)} .
$$

\section{APPENDIX C: THE MATCHED-FILTER OUTPUT FOR THE LFM CASE}

Calculations necessary to obtain Eqs. (20) and (25) are explained in this appendix. First, recall expression (17) defining the result of the matched-filter output for any signal:

$$
R(\tau, v)=\sum_{i} a_{i}\left(\eta \eta_{i}\right)^{1 / 2} \int_{-\infty}^{\infty} e\left(\eta_{i}\left(t+\tau-\tau_{i}\right)\right) e^{T}(\eta t) d t,
$$

where $e(t)$ is a LFM signal with known parameters defined in Sec. III as

$$
e(t)=\operatorname{rect}\left(\frac{t}{T}\right) \frac{1}{\sqrt{T}} \exp \left(j 2 \pi\left(f_{c} t+\frac{k}{2} t^{2}\right)\right) .
$$

We introduce the substitution

$$
t^{\prime}=t+\frac{\Delta \tau_{i}}{2}
$$

to get a symmetric expression in $\Delta \tau_{i}$ which avoids the need to consider the two cases $\Delta \tau_{i}$ positive and $\Delta \tau_{i}$ negative. Relation $(\mathrm{C} 1)$ becomes

$R(\tau, v)=\sum_{i} a_{i}\left(\eta_{i} \eta\right)^{1 / 2} \int_{-\infty}^{\infty} e\left(\eta_{i}\left(t+\frac{\Delta \tau_{i}}{2}\right)\right) e^{T}\left(\eta\left(t-\frac{\Delta \tau_{i}}{2}\right)\right) d t$.

After some manipulations with Eqs. (C2) and (C4) we obtain

$R(\tau, v)=\sum_{i} C_{i} D_{i} E_{i} \int_{-\infty}^{\infty} R_{1} R_{2} \exp \left(\frac{j \pi \gamma}{2}\left(\frac{\gamma \alpha_{i}}{\sqrt{\left|\beta_{i}\right|}}+2 t \sqrt{\left|\beta_{i}\right|}\right)^{2}\right) d t$,

where

$$
\begin{aligned}
& R_{1}=\operatorname{rect}\left(\frac{\eta_{i}\left(t+\frac{\Delta \tau_{i}}{2}\right)}{T}\right), \\
& R_{2}=\operatorname{rect}\left(\frac{\eta\left(t-\frac{\Delta \tau_{i}}{2}\right)}{T}\right),
\end{aligned}
$$

The variables $C_{i}, D_{i}, \gamma_{i}, \alpha_{i}$, and $\beta_{i}$ are introduced in Sec. III by relations (20). The bounds of integration of Eq. (C5) depend on $R_{1}$ and $R_{2}$. They are called $t_{1}$ and $t_{2}$, and Appendix $\mathrm{D}$ explains how they are obtained. Equation (C5) is reformulated with the following change in variables:

$$
X=\frac{\gamma \alpha_{i}}{\sqrt{\left|\beta_{i}\right|}+2 t \sqrt{\left|\beta_{i}\right|}},
$$

leading to

$$
R(\tau, v)=\sum_{i} \frac{C_{i} D_{i} E_{i}}{2 \sqrt{\left|\beta_{i}\right|}} \int_{X_{i}}^{Y_{i}} \exp \left(\frac{j \gamma \pi}{2} t^{2}\right),
$$

where $X_{i}$ and $Y_{i}$ can be expressed as function of $t_{1}$ and $t_{2}$ according to the change in variables defined in formula (C8),

$$
X_{i}=\frac{\gamma \alpha_{i}}{\sqrt{\left|\beta_{i}\right|}+2 t_{1} \sqrt{\left|\beta_{i}\right|}},
$$


TABLE II. Integration bounds of expression (C5).

\begin{tabular}{ccc}
\hline \hline Range of $\Delta \tau_{i}$ & $t_{1}$ & $t_{2}$ \\
\hline$\frac{-T}{2}\left(\frac{\eta_{i}+\eta}{\eta_{i} \eta}\right) \leq \Delta \tau_{i}<\frac{-T}{2}\left|\frac{\eta_{i}-\eta}{\eta_{i} \eta}\right|$ & $\frac{-\Delta \tau_{i}}{2}-\frac{T}{2 \eta}$ & $\frac{\Delta \tau_{i}}{2}-\frac{T}{2 \eta_{i}}$ \\
$\frac{-T}{2}\left|\frac{\eta_{i}-\eta}{\eta_{i} \eta}\right| \leq \Delta \tau_{i} \leq \frac{T}{2}\left|\frac{\eta_{i}-\eta}{\eta_{i} \eta}\right|$ & $\frac{-\Delta \tau_{i}}{2}-\frac{T}{2 \eta}$ & $\frac{\Delta \tau_{i}}{2}+\frac{T}{2 \eta}$ \\
$\frac{-T}{2}\left|\frac{\eta_{i}-\eta}{\eta_{i} \eta}\right| \leq \Delta \tau_{i} \leq \frac{T}{2}\left|\frac{\eta_{i}-\eta}{\eta_{i} \eta}\right|$ & $\frac{\Delta \tau_{i}}{2}-\frac{T}{2 \eta_{i}}$ & $\frac{\Delta \tau_{i}}{2}+\frac{T}{2 \eta_{i}}$ \\
and $\eta_{i}>\eta$ & $\frac{\Delta \tau_{i}}{2}-\frac{T}{2 \eta_{i}}$ & $\frac{-\Delta \tau_{i}}{2}+\frac{T}{2 \eta}$ \\
\hline$\frac{\eta_{i}-\eta}{2} \mid \frac{\eta_{i} \eta}{2}$ & & $\frac{\eta_{i}+\eta}{\eta_{i} \eta} \mid$
\end{tabular}

$$
X_{i}=\frac{\gamma \alpha_{i}}{\sqrt{\left|\beta_{i}\right|}+2 t_{2} \sqrt{\left|\beta_{i}\right|}} .
$$

Finally, the result of Eq. (C9) can be expressed and simplified with a complex form of the Fresnel integrals if $v$ is different from $v_{i}$,

$$
R(\tau, v)=\sum_{i} \frac{C_{i} D_{i} E_{i}}{2 \sqrt{\left|\beta_{i}\right|}}\left(F\left(Y_{i}\right)-F\left(X_{i}\right)\right),
$$

where

$$
\begin{aligned}
& F(u)=C(u)+j \gamma S(u), \\
& C(u)=\int_{0}^{u} \cos \left(\frac{\pi t^{2}}{2}\right) d t, \\
& S(u)=\int_{0}^{u} \sin \left(\frac{\pi t^{2}}{2}\right) d t .
\end{aligned}
$$

When the Doppler transformation of the reference signal matches exactly the Doppler transformation of the $i$ th path, Eq. (21) is no longer valid. Equation (C5) becomes

$$
R(\tau, u)=\sum_{i} C_{i} \int_{t_{1}}^{t_{2}} \exp \left(2 j \pi t k \Delta \tau_{i} \eta^{2}\right) d t,
$$

where bounds of integration are given by $R_{1}$ and $R_{2}$, and when $\eta_{i}=\eta$ they satisfy

$$
\begin{aligned}
& t_{1}=\frac{\left|\Delta \tau_{i}\right|}{2}-\frac{T}{2 \eta}, \\
& t_{2}=\frac{T}{2 \eta}-\frac{\left|\Delta \tau_{i}\right|}{2}, \\
& \left|\Delta \tau_{i}\right| \leq \frac{T}{\eta} .
\end{aligned}
$$

The integration of formula (C16) finally gives the expression of the output matched-filter when Doppler transformation of the reference signal matches exactly with the Doppler transformation of the $i$ th path

$$
R(\tau, v)=\sum_{i} C_{i}\left(\left|\Delta \tau_{i}\right|-\frac{T}{\eta_{i}}\right) \frac{\sin \left(\xi_{i}\right)}{\xi_{i}},
$$

where

$$
\xi_{i}=\pi k \Delta \tau_{i} \eta_{i}\left(\eta_{i}\left|\Delta \tau_{i}\right|-T\right) .
$$

\section{APPENDIX D: DETERMINATION OF THE INTEGRATION BOUNDS}

The bounds of integration of expression (C5) are called $t_{1}$ and $t_{2}$ and are given by $R_{1}$ and $R_{2}$. Different cases appear depending on both the length and the position of $R_{1}$ compared with $R_{2}$. When $\eta_{i}$ is different from $\eta$, there are four possible values for each bound of integration and they may be sorted by the range of $\Delta \tau_{i}$, as summarized in Table II.

${ }^{1}$ Z. H. Michalopoulou, "Matched-impulse-response processing for shallowwater localization and geoacoustic inversion," J. Acoust. Soc. Am. 108, 2082-2090 (2000).

${ }^{2}$ M. I. Taroudakis and G.-N. Makrakis, Inverse Problems in Underwater Acoustics (Springer-Verlag, New York, 2001).

${ }^{3}$ A. Baggeroer, W. Kuperman, and P. Mikhalevsky, "An overview of matched field methods in ocean acoustics," IEEE J. Ocean. Eng. 18, 401424 (1993).

${ }^{4}$ C. Gervaise, S. Vallez, Y. Stephan, and Y. Simard, "Robust 2d localization of low-frequency calls in shallow waters using modal propagation modelling," Can. Acoust. 36, 153-159 (2008).

${ }^{5}$ C. Gervaise, S. Vallez, C. Ioana, Y. Stephan, and Y. Simard, "Passive acoustic tomography: New concepts and applications using marine mammals: A review," J. Mar. Biol. Assoc. U.K. 87, 5-10 (2007).

${ }^{6}$ S. Qian and D. Chen, "Signal representation using adaptive normalized Gaussian functions," Signal Process. 36, 1-11 (1994).

${ }^{7}$ S. Mallat and Z. Zhang, "Matching pursuits with time-frequency dictionaries," IEEE Trans. Signal Process. 41, 3397-3415 (1993).

${ }^{8}$ H. Zou, Y. Chen, J. Zhu, Q. Dai, G. Wu, and Y. Li, "Steady-motion-based Dopplerlet transform: Application to the estimation of range and speed of a moving sound source," IEEE J. Ocean. Eng. 29, 887-905 (2004).

${ }^{9}$ A. N. Guthrie, R. M. Fitzgerald, D. A. Nutile, and J. D. Shaffer, "Longrange low-frequency cw propagation in the deep ocean: AntiguaNewfoundland," J. Acoust. Soc. Am. 56, 58-69 (1974).

${ }^{10}$ K. E. Hawker, "A normal mode theory of acoustic Doppler effects in the oceanic waveguide," J. Acoust. Soc. Am. 65, 675-681 (1979).

${ }^{11}$ P. H. Lim and J. M. Ozard, "On the underwater acoustic field of a moving point source. I. Range-independent environment," J. Acoust. Soc. Am. 95, 131-137 (1994).

${ }^{12}$ R. P. Flanagan, N. L. Weinberg, and J. G. Clark, "Coherent analysis of ray propagation with moving source and fixed receiver," J. Acoust. Soc. Am. 56, 1673-1680 (1974).

${ }^{13}$ J. G. Clark, R. P. Flanagan, and N. L. Weinberg, "Multipath acoustic propagation with a moving source in a bounded deep ocean channel," J. Acoust. Soc. Am. 60, 1274-1284 (1976).

${ }^{14}$ J. P. Hermand and W. I. Roderick, "Delay-Doppler resolution performance of large time-bandwidth-product linear fm signals in a multipath ocean environment," J. Acoust. Soc. Am. 84, 1709-1727 (1988).

${ }^{15} \mathrm{C}$. L. Pekeris, "Theory of propagation of explosive sound in shallow water," Propagation of Sound in the Ocean (Geological Society of America, New York, 1948), Memoir 27, pp. 1-117.

${ }^{16}$ F. B. Jensen, W. A. Kuperman, and H. Schmidt, Computational Ocean Acoustics (AIP, New York, 1994).

${ }^{17}$ S. Kramer, "Doppler and acceleration tolerances of high-gain, wideband linear fm correlation sonars," Proc. IEEE 55, 627-636 (1967).

${ }^{18}$ W. Adams, J. Kuhn, and W. Whyland, "Correlator compensation requirements for passive time-delay estimation with moving source or receivers," IEEE Trans. Acoust., Speech, Signal Process. 28, 158-168 (1980).

${ }^{19}$ B. Harris and S. Kramer, "Asymptotic evaluation of the ambiguity functions of high-gain fm matched filter sonar systems," Proc. IEEE 56, 21492157 (1968).

${ }^{20}$ N. F. Josso, C. Ioana, C. Gervaise, Y. Stephan, and J. I. Mars, "Motion effect modeling in multipath configuration using warping based lagDoppler filtering," IEEE Trans. Acoust., Speech, Signal Process. 2009, 
2301-2304

${ }^{21} \mathrm{G}$. Theuillon and Y. Stephan, "Geoacoustic characterization of the seafloor from a subbottom profiler applied to the BASE'07 experiment," J. Acoust. Soc. Am. 123, 3108 (2008).

${ }^{22}$ N. Josso, C. Ioana, C. Gervaise, and J. I. Mars, "On the consideration of motion effects in underwater geoacoustic inversion," J. Acoust. Soc. Am. 123, 3625 (2008).

${ }^{23}$ N. F. Josso, C. Ioana, J. I. Mars, C. Gervaise, and Y. Stephan, "Warping based lag-Doppler filtering applied to motion effect compensation in acoustical multipath propagation," J. Acoust. Soc. Am. 125, 2541 (2009). 